\title{
Network Structure of a System coded by Groups
}

\author{
H. G. Busse and B. H. Havsteen \\ Biochemisches Institut im Fachbereich Medizin, Universität Kiel
}

Z. Naturforsch. 35a, 526-530 (1980); received February 15, 1980

\begin{abstract}
A system may be characterized by its dynamics as well as by its structure. Several kinds of schematic drawings have been proposed to represent the structure of a system. Here, the representation of the structure by free groups is discussed. Free groups are an abstract mathematical tool, which permits the systematic treatment of a structure. The suggestion is exemplified by its application to a chemical kinetic model. The structure of the model is given (coded) by a linear sequence of symbols. The interaction of the environment with the structure (mutation) and the relation between structure and systemic boundaries (finiteness) are treated. The concept probably also applies to biological systems. Also, context-free languages could be used as alternate representations of system structures.
\end{abstract}

\section{Introduction}

Most of the natural systems consist of a huge number of interacting components. A common trait of these systems is that they possess a structure, which contains the information about how the components are assembled and dynamics which are related to the constitutive laws of each component. By a television set, this aspect is evident since the structure of its electronics is defined by a circuit diagram and its dynamics is represented by the time course of all currents and voltages which operate in the set. Moreover, the constitutive laws of the components may be recognized, e.g. as Ohm's law for resistors or Henry's law for inductors. Electronic circuits, even if they exhibit the complexity of a computer, belong to a class of systems which is governed by the thermodynamics of irreversible processes. This theory comprises all physical systems which obey the laws of classical physics, e.g. Maxwell's laws of electromagnetism and the laws of mechanics, including hydro- and aerodynamics as well as gravitational mechanics. Also, chemical kinetics [1] belongs to this class of system. Hence, the dynamics in chemical reaction systems may be represented in this way. The systems in biology, sociology, and economics are probably also of the same kind, but their complexity so far prevented a detailed analysis.

Reprint request to Dr. H. G. Busse, Biochemisches Institut im Fachbereich Medizin der Universität Kiel, Olshausenstraße 40-60, Gebäude N 11, D-2300 Kiel 1.
Here, we shall treat a kinetic model [2] system, which admittedly is somewhat artificial but has the advantage of being mathematically tractable. The essential feature exhibited by the model is the property that its structure may be represented by an infinite abelean group. Moreover, the particular representation of the group permits the coding of the structure in a linear sequence, like that of a printed message. Furthermore, the model permits the distinction between the system itself and its environment. Hence, the interaction of the environment with the system, in particular with its structure, can be studied. Such an interaction could in biology be interpreted as survival by mutational adaption to the environment.

\section{Model System}

Dreitlein and Smoes [2] have interpreted a particular system of differential equations [3] in terms of chemical kinetics. The generalized differential equation system of the autonomous type:

$$
\begin{aligned}
& \frac{\mathrm{d}}{\mathrm{d} t} a_{1}=\left(e-a_{1}{ }^{2}-a_{2}{ }^{2}\right) a_{1}+a a_{2}-2 c a_{1}, \\
& \frac{\mathrm{d}}{\mathrm{d} t} a_{2}=\left(e-a_{1}{ }^{2}-a_{2}{ }^{2}\right) a_{2}-b a_{1}+2 c a_{2},
\end{aligned}
$$

where $e, a, b$, and $c$ are constant parameters, may be transformed by $x=a_{1}+q ; y=a_{2}+p$ to a system, which is acceptable in chemical kinetics:

$$
\begin{aligned}
\frac{\mathrm{d}}{\mathrm{d} t} x=v_{0} & +\left(e-2 c-3 q^{2}-p^{2}\right) x+3 q x^{2}-x^{3} \\
& +(a-2 q p) y+q y^{2}+2 p x y-x y^{2} \\
& +q^{3}+q p^{2}
\end{aligned}
$$

0340-4811/80/0500-0526 \$01.00/0. - Please order a reprint rather than making your own copy. 


$$
\begin{aligned}
\frac{\mathrm{d}}{\mathrm{d} t} y=w_{0} & +\left(e+2 c-3 p^{2}-q^{2}\right) y+3 p y^{2}-y^{3} \\
& -(b+2 p q) x+p x^{2}+2 q x y-x^{2} y+p^{3} \\
& +p q^{2}
\end{aligned}
$$

where $v_{0}=2 c q-e q-a p$ and $w_{0}=-2 c p-e p+$ $b q ; p, q$ are constant parameters.

The Eqs. (2) must correspond to a chemical reaction mechanism to be the rate equations of its chemical kinetics. Dreitlein and Smoes [2] have established a mechanistic scheme of reactions, which would result in Equations (2). Table 1 gives the slightly modified scheme. In particular Eqs. $(3 \mathrm{a})$ and (8 a) [2] have been contracted to one reaction, so that $\mathrm{X}$ and $\mathrm{Y}$ now are coupled and the reverse reaction has been added to $(1 \mathrm{~b})$ and $(6 \mathrm{~b})$. If the catalytic compounds are neglected and compound $\mathrm{H}$ is $\mathrm{C}, \mathrm{U}$ is $\mathrm{P}, \mathrm{L}$ is $\mathrm{B}, \mathrm{D}$ is $\mathrm{F}, \mathrm{O}$ is $\mathrm{Q}$, and $\mathrm{N}$ is $\mathrm{Z}$, then a simple overall scheme results:

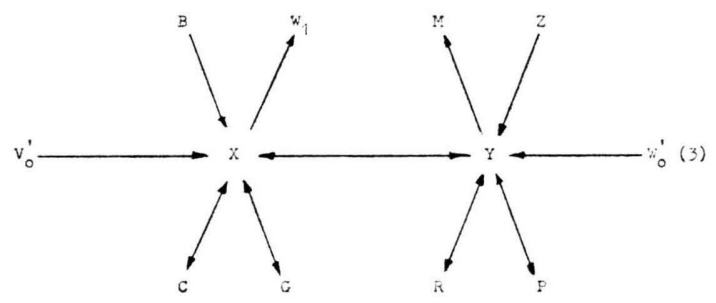

which illustrates the flow of chemicals in and out of the substances considered, $\mathrm{X}$ and $\mathrm{Y}$. Some of these reactions are treated as if they were truly irreversibly which is incompatible with reality. However, one may add the neglected terms to Eqs. (2) and furnish them with factors, e. g. $\varepsilon$ and $\mu$, resp., which are so small that they hardly influence the dynamics of the system. Hence, Eqs. (2) acquire the form:

$$
\begin{aligned}
\frac{\mathrm{d} x}{\mathrm{~d} t}=v_{0} & +\cdots+\varepsilon\left\{-k_{-1 a} x^{2}+k_{-3 b} y W_{1} W\right. \\
& \left.+k_{-5} x^{2} y\right\} \\
\frac{\mathrm{d} y}{\mathrm{~d} t}=w_{0} & +\cdots+\mu\left\{-k_{-6 a} y^{2}+k_{-8 b} x M V\right. \\
& \left.+k_{-10} y^{2} x\right\}
\end{aligned}
$$

where...represents terms already listed in Equations (2).

Thus, a model system has been developed for which the structure may be represented by group relations.

\section{Group Relations}

A group, finite or infinite, may be represented [4] by a set of generators and a set of relations. This description of a structure is suitable to formulate the reaction mechanisms [5] in Table 1 as a group. The generators are the chemical compounds. From the graph (3), the set of generators:

$$
\{\mathrm{X}, \mathrm{Y}, \mathrm{B}, \mathrm{C}, \mathrm{G}, \mathrm{W}, \mathrm{M}, \mathrm{Z}, \mathrm{R}, \mathrm{P}\}
$$

is derived. The relations are the reactions. Since the representation of the reactions in Table 1 is not elementary, the dynamic aspects are sacrificed by such a representation. However, if the real elementary steps of the mechanism are used, then the true dynamics are described. Hence, any chemical reaction may be represented by an equation of the type:

$$
\mathrm{A}+\mathrm{B} \rightleftharpoons \mathrm{C},
$$

where $\mathrm{A}, \mathrm{B}, \mathrm{C}$ are the chemical compounds, i. e. the generators, and the group relation:

$$
\mathrm{ABC}^{-1}=\mathrm{E} \text {, }
$$

where $\mathrm{E}$, the unity element of the group, uniquely represents the reaction (5), if $\mathrm{A}, \mathrm{B}$ and $\mathrm{C}$ are independent generators, i.e. if the inverses of the generators are not included in the set of generators. Hence, any proper triple of the form (6) gives a possible reaction if the generators $\mathrm{A}, \mathrm{B}$ and $\mathrm{C}$ are suitably chosen from the set of generators (4). An inproper triple would be obtained if, e. g. $\mathrm{C}$ is equal to $\mathrm{B}$. Then, the relation $\mathrm{ABB}^{-1}=\mathrm{E}$, i. e. $\mathrm{A}=\mathrm{E}$, would result, which classifies $\mathrm{A}$ as not belonging to the set of proper generators. $\mathrm{Bi}$ - and trimolecular reactions may easily be converted to reactions of the type (5) by postulating intermediate reactants. Hence, reaction ( 1 a) of Table 1 may be recast as:

$$
\mathrm{B}+\mathrm{X} \rightarrow \mathrm{B}^{\prime} \rightarrow \mathrm{X}+\mathrm{X}
$$

to give the relations $\mathrm{BXB}^{\prime-1}=\mathrm{E}$ and $\mathrm{XXB}^{-1}=\mathrm{E}$.

In an abbreviated form, the equal sign may be omitted, whence the relations may be written in a compact form as:

$$
\mathrm{BXB}^{\prime-1} \mathrm{EXXB}^{-1} \mathrm{E} \text {, }
$$

where the unity element of the group acts as a separator. By this method, all reactions in Table 1 , which imply the condition $p>0$ or $q>0$, may be represented by a string of symbols of the kind (7). The remaining reactions depend on the conditions 
Table 1. Listing of chemical reactions which lead to the rate Equations [2]. The column ,,condition" gives the restrictions which originate from the positive, definite character of the concentrations and the rate constant.

\begin{tabular}{|c|c|c|c|c|c|}
\hline No & Reaction & & Rate law & & Condition \\
\hline $1 \mathrm{a}$ & $B+X$ & $\rightarrow \mathrm{X}+\mathrm{X}$ & $k_{1 \mathrm{a}}[\mathrm{B}]$ & $\begin{array}{l}=e-2 d \\
=0\end{array}$ & $\begin{aligned} & e-2 d \geq 0 \\
& \leqq 0\end{aligned}$ \\
\hline b & $\mathrm{D}+\mathrm{X}$ & $\rightleftharpoons \mathrm{F}+\mathrm{G}$ & $\begin{array}{l}k_{1 \mathrm{~b}}[\mathrm{D}] \\
k_{-1 \mathrm{~b}}[\mathrm{~F}][\mathrm{G}]\end{array}$ & $\begin{array}{l}=3 q^{2}+p^{2} \\
=3 q^{2}+p^{2}+|e-2 d| \\
=q^{3}+q p^{2}\end{array}$ & $\begin{aligned} & \geqq 0 \\
q & >0\end{aligned}$ \\
\hline 2 & $3 \mathrm{X}$ & $\rightleftharpoons 2 \mathrm{X}+\mathrm{C}$ & $k_{2 \mathrm{a}}=1 ; k_{-2 \mathrm{a}}[\mathrm{C}]$ & $=3 q$ & $q>0$ \\
\hline $3 \mathrm{a}$ & $\mathrm{Y}+\mathrm{S}$ & $\rightarrow \mathrm{X}+\mathrm{S}$ & $k_{3 \mathrm{a}}[\mathrm{S}]$ & $\begin{array}{l}=a \\
=0\end{array}$ & $\begin{aligned} a & >0 \\
& <0\end{aligned}$ \\
\hline b & $\mathrm{Y}+\underset{\mathrm{J}}{\mathrm{J}}$ & $\begin{array}{l}\rightarrow \mathrm{Y}+\mathrm{W}_{1}+\mathrm{W} \\
\rightleftharpoons \mathrm{X}+\mathrm{W}\end{array}$ & $k_{3 \mathrm{~b}}[\mathrm{~J}]$ & $\begin{array}{l}=2 q p \\
=2 q p+|a|\end{array}$ & $\begin{array}{l}>0 \\
<0\end{array}$ \\
\hline 4 & $X+2 Y$ & $\rightleftharpoons 2 \mathrm{Y}+\mathrm{H}$ & $k_{4}=1 ; k_{-4}[\mathrm{H}]$ & $=q$ & $q>0$ \\
\hline 5 & $\mathrm{~L}+\mathrm{X}+$ & $Y \rightarrow 2 X+Y$ & $k_{5}[\mathrm{~L}]$ & $=2 p$ & $p>0$ \\
\hline $6 \mathrm{a}$ & $\mathrm{N}+\mathrm{Y}$ & $\rightarrow \mathrm{Y}+\mathrm{Y}$ & $k_{6 \mathrm{a}}[\mathrm{N}]$ & $\begin{array}{l}=e+2 d \\
=0\end{array}$ & $\begin{aligned} e+2 d & \geqq 0 \\
& \leqq 0\end{aligned}$ \\
\hline b & $Q+Y$ & $\rightleftharpoons \mathrm{R}+0$ & $\begin{array}{l}k_{6 \mathrm{~b}}[\mathrm{Q}] \\
k_{-6 \mathrm{~b}}[\mathrm{R}][0]\end{array}$ & $\begin{array}{l}=3 p^{2}+q^{2} \\
=3 p^{2}+q^{2}+|e+2 d| \\
=p^{3}+p q^{2}\end{array}$ & $\begin{aligned} & \geqq 0 \\
p & >0\end{aligned}$ \\
\hline 7 & $3 \mathrm{Y}$ & $\rightleftharpoons 2 \mathrm{Y}+\mathrm{U}$ & $k_{7}=1 ; k_{-7}[\mathrm{U}]$ & $=3 p$ & $p>0$ \\
\hline $8 \mathrm{a}$ & $\mathrm{X}+\mathrm{S}$ & $\rightarrow \mathrm{Y}+\mathrm{S}$ & $k_{-3 \mathrm{a}}[\mathrm{S}]$ & $\begin{array}{l}=+|b| \\
=0\end{array}$ & $\begin{aligned} b & <0 \\
& >0\end{aligned}$ \\
\hline b & $\mathrm{X}+\underset{\mathrm{P}}{\mathrm{P}}$ & $\begin{array}{l}\rightarrow \mathrm{X}+\mathrm{M}+\mathrm{V} \\
\rightleftharpoons \mathrm{Y}+\mathrm{V}\end{array}$ & $k_{8 \mathrm{~b}}[\mathrm{P}]$ & $\begin{array}{l}=2 p q+|b| \\
=2 p q\end{array}$ & $\begin{array}{l}>0 \\
<0\end{array}$ \\
\hline 9 & $\mathrm{Y}+2 \mathrm{X}$ & $\rightleftharpoons 2 \mathrm{X}+\mathrm{P}$ & $k_{9}=1 ; k_{-9}[\mathrm{P}]$ & $=p$ & $p>0$ \\
\hline 10 & $Z+X+$ & $Y \rightarrow X+2 Y$ & $k_{10}[\mathrm{Z}]$ & $=2 q$ & $q>0$ \\
\hline
\end{tabular}

of the parameters, $a, b, e$ and $c$. In reactions $1 \mathrm{~b}$, $6 \mathrm{~b}, 3 \mathrm{~b}$ and $8 \mathrm{~b}$, the reaction rate changes according to the rate relations given in Table 1 . However, the reactions 1 a, 3 a, 6 a and 8 a may be rendered completely inactive under certain conditions (see Table 1). Hence, ranges of the parameters exist, in which some of the reactions may be omitted from the reaction scheme without effecting the dynamics. In Table 2, the active reaction sequences have been summarized for the different parameter ranges.

Table 2. Chemical reaction sequences for different parameter conditions.

\begin{tabular}{|c|c|c|c|c|c|}
\hline \multicolumn{2}{|c|}{$\begin{array}{l}e-2 d \\
e+2 d\end{array}$} & \multirow{2}{*}{$\begin{array}{l}>0 \\
>0 \\
\bar{B} \overline{\mathrm{N}} \overline{\mathrm{S}}\end{array}$} & \multirow{2}{*}{ 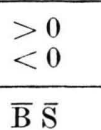 } & \multirow{2}{*}{$\begin{array}{l}<0 \\
>0 \\
\bar{N} \bar{S}\end{array}$} & \multirow{2}{*}{$\begin{array}{l}<0 \\
<0 \\
\overline{\mathrm{S}}\end{array}$} \\
\hline$>0$ & $>0$ & & & & \\
\hline$>0$ & $<0$ & $\overline{\mathrm{B}} \overline{\mathrm{N}} \overline{\mathrm{S}} \overline{\mathrm{R}}$ & $\overline{\mathrm{B}} \overline{\mathrm{S}} \overline{\mathrm{R}}$ & $\overline{\mathrm{N}} \overline{\mathrm{S}} \overline{\mathrm{R}}$ & $\overline{\mathrm{S}} \overline{\mathrm{R}}$ \\
\hline$<0$ & $>0$ & $\overline{\mathrm{B}} \overline{\mathrm{N}}$ & & & - \\
\hline$\underset{\mathrm{a}}{<0}$ & $\underset{\mathrm{b}}{<0}$ & $\overline{\mathrm{B}} \overline{\mathrm{N}} \overline{\mathrm{R}}$ & $\bar{B} \bar{R}$ & $\overline{\mathrm{N}} \overline{\mathrm{R}}$ & $\overline{\mathrm{R}}$ \\
\hline
\end{tabular}

$\overline{\mathrm{B}}=\mathrm{BX}_{\mathrm{B}^{\prime}}-1 \mathrm{EXX}^{\prime} \overline{\mathrm{B}}^{\prime}-1 \mathrm{E} ; \overline{\mathrm{N}}=\mathrm{NY} \overline{\mathrm{N}}^{\prime}-1 \mathrm{EYY} \overline{\mathrm{N}}^{\prime}-1 \mathrm{E} ;$

$\overline{\mathrm{S}}=\mathrm{YSS}^{\prime}-1 \mathrm{E} ; \overline{\mathrm{R}}=\mathrm{XSS}^{\mathrm{S}^{\prime}-1} \mathrm{E}$.
Thus, the mechanism depends on the set of parameters.

Besides, the representation of the structure of the system by a linear sequence of symbols, i. e. the group representation, reveals another property inherent in the structure. It limits the real range of system states by boundaries. Differential operators [6] exist for abelean groups, to which the discussed chemical systems belong. A differential operator - the boundary operator - may be obtained from the relations of the group. How this is achieved, will not be outlined here since the procedure has already been published [7] in detail. However, the meaning of this operator should be explained. The mass conservation law of the chemical system is achieved by the boundary operator, i. e. by the conservation of elements or charges. These laws restrict the range of concentrations acceptable to the system as soon as initial conditions have been given. Note, that the restrictions are independent of the constitutive laws of kinetics (i. e. of mono- or bimolecular laws of reaction). Usually, some rela- 
tions are not listed as group relations, since they may be part of the group axioms and therefore always are valid. The relations permit the existence of the inverse of any generator, e.g. $\mathrm{AA}^{-1}=\mathrm{E}$ where $\mathrm{A}$ is a generator of the set. The definition may be interpreted as a boundary operation related to the positive, definite values of the concentrations. Hence, the relations describe a limited space in the space of concentrations. The system can only move within this closed volume. Note, that the actual motion only is defined if the kinetic laws are known, i. e. again, the volume restriction is independent of the laws of motion but dependent on the initial condition. The structure inherent in the group subdivides the space because it restricts the motion of the system.

Furthermore, the network structure [7] of the system may be derived from the boundary operator. In an electronic system, the structure is known as the flow diagram, in some other systems as the equivalent circuit and for chemical systems, the structure is sometimes called the thermodynamic network. Hence, the representation of the system structure by group relations is another way of describing the structural connections in a system. However, it is superior to the other methods mentioned because it uses a mathematically well defined technique for the representation and because extensions and manipulations of the system can be studied more easily. Chemical and thermodynamic systems demand that the group is abelean. This restriction may not be fulfilled by some other complex systems since commutation of generators is not always permitted. Such extensions or restrictions will become apparent in the linear sequence coding of the relations of the group (see (7)). Note, that the abelean property of a group also can be expressed in relations [4].

However, there is another remarkable representation of structures admitted by groups by the mathematical technique of context-free languages. The need for computer languages has caused a rapid development of this field. In contrast to the representations which have been used in this paper, here the monoid (a semigroup with a neutral element) forms the basic element. Any group is a monoid. Hence, the theory of context-free languages may also be used for formulate structures. Context-free languages consist of an alphabet $\mathrm{H}$, a set of variables $\mathrm{V}$ and a set of productions $\mathrm{P}$ which are summarized in a grammar $\mathrm{G}=\langle\mathrm{V}, \mathrm{H}, \mathrm{P}\rangle$. The alphabet generates the elements (i. e. text) of the monoid (group). The rules for the construction of the elements are given by relations (productions) between $\mathrm{H}$ and $\mathrm{V}$. Thus, e. g. the expression of (5) as a production is

$$
\xi \rightarrow \mathrm{AB} \overline{\mathrm{C}},
$$

where the alphauet is the union of the sets $\{A, B\}$ $\mathrm{U}\{\overline{\mathrm{C}}\}$ and the variables are the set $\{\xi\}$. Here, the alphabet is composed of sets like the one used in Dyck languages [10]. In this representation, the boundary relations appear as productions. Hence, the structure is inherent in the grammar of the language.

Context-free languages can be used to represent the structure of systems. Since they are based on monoids and productions, of which free groups and their relations are subsets, they form a generalized basis for the representation of structures.

\section{Discussion}

In the preceding sections it was shown that the structure of a dynamic system can be described by the algebraic structure of a group. Specifically, the representation of the group by generators and relations has led to the coding of the structure as a linear sequence of generators. Moreover, the model system showed that its structure may depend on the parameters in the differential equation system which describes the system. Consequently, the coding sequence can change if parameters of the system vary. The system may be furnished with an environment if $v_{0}$ and $w_{0}$ are considereed fluxes from the environment into the system. In this case, an interesting phenomenon appears: The flux

$$
v_{0}=(2 d-e) q-a p
$$

can change under the control of the environment. If $q, p$ and a remain constant, then $2 d-e$ may change so as to validate (8). Depending on the influx $v_{0},(2 d-e)$ may take positive or negative values. From reaction 1 a of Table 1 , it follows that reaction $1 \mathrm{a}$ may in one case be switched on, whereas in another it is totally annihilated. Hence, it can, under the condition $2 d-e<0$, be omitted from the mechanism without effect on the dynamics (see Table 2). Here, the environment is mapped onto the structure of the system. Therefore, there is a coupling between the environment and the structure of the system, a phenomenon which is well 
known from biological systems. There are other striking similarities to biological systems, e.g. the coding of the structure in a linear sequence, in which the structural elements are separated by spacers (separating symbol). Moreover, Table 2 may be considered as a mutation map because, under specific environmental conditions, a gene (reaction) may appear to be missing from the linear sequence of the structure of the system. Thus, the system can adapt to the environment, i. e. the constraints by the system parameters. Even if the model treated in this respect is not ideal and not a real chemical reaction system, it may serve as a useful example. Models which are more suitable for the discussion of the interaction between the structure of a system, its dynamics and environment may perhaps be constructed.

The ambiguity in the formulation of chemical reaction mechanisms is reflected in the representation of its free group. In a mechanism, several steps may be summarized in one overall reaction. The same is true for group relations. This ambiguity sometimes renders the recognition of identical group structures difficult in relations which appear to be unrelated. The generator set of the group may also be altered by addition of new group elements.

The boundary formation seems to be a basic property inherent in physical systems. Here, it is referred to the structure of the system. However, the boundary operators span a vector space [8]

[1] G. F. Oster, A. S. Perelson, and A. Katchalsky, Quart. Rev. Biophys. 6, 1 (1973).

[2] J. Dreitlein and M. L. Smoes, J. Theor. Biol. 46, 559 (1974).

[3] A. Cohen, An Introduction to the Lie Theory, Heath \& Co., Boston 1911, p.235.

[4] H. Seiffert and W. Threlfall, Lehrbuch der Topologie, Chelsea Publ. Co., New York 1947.

[5] P. H. Sellers, Arch Rational Mech. Anal. 44, 23 (1971).

[6] S. M. Lane, Homology, Grundl. Math. Wiss., Bd. 114, Springer-Verlag, Berlin 1963.

[7] A. S. Perelson and G. F. Oster, Arch. Rational Mech. Anal. 57, 31 (1979). and hence define the dual and tensor spaces [9]. Thus, the structure of the system possesses all the properties of vector spaces. The essential prerequisite for a basis of a vector space is the com. mutativity of the basis elements. This is characteristic for an abelean group. Since the relations which render a group abelean can be added to the relation which defines a group, any group can be assigned to an abelean group (possibly a trivial one) and consequently to a vector space.

In summary, the aim of this paper is to demonstrate the usefulness of the representation of the structure of a system by free groups. It also emphasizes that the structure of the system is the feature, which normally contains the most essential information. This information can be coded by a linear sequence. If the sequence of the code is a part of the system, then the latter may be self-reproductive. The interaction between structure and environment of the system could probably be analyzed in more detail by this representation than by the ones which previously have been used.

The context-free languages [11] may be a very useful tool to study the retrieval and translation of structural information which is stored in biological systems. In this connection, the mathematical definition of transducers and of automatons is of particular interest since biological information processes, e.g. in biosynthesis, show very similar characteristics.

[8] C. v. Westenholz, Ann. Inst. Henri Poincaré 15, 189 (1971).

[9] M. Spivak, Calculus on Manifolds, Benjamin, New York 1965.

[10] W. Magnus, A. Karacs, and D. Solitar, Combinatorial Group Theory, Inters. Publ. New York 1966.

M. P. Schützenberger, in: P. Suppes et al (ed.), Logic Methodology and Philosophy of Science IV, Am. Elsevier, New York 1973, p. 197.

[11] S. Eilenberg, Automata, Languages, and Machines, Vol. C, Acad. Press, New York 1978. 